\title{
Article
}

\section{Combined Effects from Solvation and Nuclear Quantum Fluctuations on Autoionization Mechanisms in Aqueous Clusters}

\author{
Laszlo Turi, Javier Rodriguez, and Daniel H. Laria
}

J. Phys. Chem. B, Just Accepted Manuscript • DOI: 10.1021/acs.jpcb.9b11087 • Publication Date (Web): 19 Feb 2020

Downloaded from pubs.acs.org on February 28, 2020

\section{Just Accepted}

"Just Accepted" manuscripts have been peer-reviewed and accepted for publication. They are posted online prior to technical editing, formatting for publication and author proofing. The American Chemical Society provides "Just Accepted" as a service to the research community to expedite the dissemination of scientific material as soon as possible after acceptance. "Just Accepted" manuscripts appear in full in PDF format accompanied by an HTML abstract. "Just Accepted" manuscripts have been fully peer reviewed, but should not be considered the official version of record. They are citable by the Digital Object Identifier (DOI@). "Just Accepted" is an optional service offered to authors. Therefore, the "Just Accepted" Web site may not include all articles that will be published in the journal. After a manuscript is technically edited and formatted, it will be removed from the "Just Accepted" Web site and published as an ASAP article. Note that technical editing may introduce minor changes to the manuscript text and/or graphics which could affect content, and all legal disclaimers and ethical guidelines that apply to the journal pertain. ACS cannot be held responsible for errors or consequences arising from the use of information contained in these "Just Accepted" manuscripts. 


\title{
Combined Effects from Solvation and Nuclear
}

\section{Quantum Fluctuations on Autoionization}

\section{Mechanisms in Aqueous Clusters}

\author{
Lászlo Túri, ${ }^{\dagger}$ Javier Rodriguez, ${ }^{\ddagger}$ and Daniel Laria*,‡ \\ Institute of Chemistry, Eötvös Loránd University \\ P. O. Box 32, Budapest, 112 H-1518, Hungary., Departamento de Física de la Materia \\ Condensada, Comisión Nacional de Energía Atómica, Avenida Libertador 8250. 1429 Buenos \\ Aires, Argentina, ECyT, UNSAM, Martín de Irigoyen 3100. 1650 San Martín. Provincia de \\ Buenos Aires, Argentina, and Departamento de Química Inorgánica, Analítica y Química-Física \\ and INQUIMAE-CONICET, Facultad de Ciencias Exactas y Naturales. Universidad de Buenos \\ Aires Ciudad Universitaria, Pabellón II. 1428 Buenos Aires - Argentina. \\ E-mail: dhlaria@cnea.gov.ar
}

\footnotetext{
${ }^{*}$ To whom correspondence should be addressed

†Eötvös Loránd University

‡Comisión Nacional de Energía Atómica

"ECyT-UNSAM

$\S$ Universidad de Buenos Aires-INQUIMAE
} 


\begin{abstract}
Using Path Integral Molecular Dynamics simulations we examine isomerization paths involving collective proton transfers in $\left[\mathrm{H}_{2} \mathrm{O}\right]_{5}$ and $\left[\mathrm{H}_{2} \mathrm{O}\right]_{8}$ clusters at cryogenic conditions. We focused attention on combined effects derived from solvation and nuclear quantum fluctuations on the characteristics of free energy barriers and relative stabilities of reactants and products. In particular, we analyzed two different processes: the first one involves the exchange of donoracceptor hydrogen bond roles along cyclic moieties, whereas the second one corresponds to charge separation leading to stable $\left[\mathrm{H}_{3} \mathrm{O}\right]^{+}[\mathrm{OH}]^{-}$ion pairs. In the first case, the explicit incorporation of quantum tunneling introduces important modifications in the classical free energy profile. The resulting quantum profile presents two main contributions, one corresponding to compressions of O-O distances, and a second one ascribed to nuclear tunneling of the light protons. Solvation effects promote a moderate polarization of the cyclic structures and a partial loss of concertedness in the collective modes, most notably, at the onset of tunneling. Still, the latter effects are also sufficiently strong to promote the stabilization of ion pairs along the classical trajectories. Contrasting, the explicit incorporation of nuclear quantum fluctuations brings charge separated configurations marginally stable. As such, the latter states could be also regarded as short-lived intermediate states along the reactive exchange path.
\end{abstract}




\section{Introduction}

The autoprotolysis of water plays a fundamental role as a controlling agent of all acid/base equilibria in aqueous solutions. For quite a long time, and despite its apparent simplicity, the microscopic interpretation of the mechanisms that lead to the proton detachment from an initially neutral water molecule and its eventual recombination remained elusive, in part, due to the lack of direct experimental validation. The advent of ab initio molecular dynamics simulations opened possibilities for significant advances in unveiling the details of the different steps involved in the molecular mechanism. At present, there seems to be sufficient evidence that rare events leading to successful autoionization processes are the results of an unlikely sequence of events. These include electric field fluctuations leading to cleavages of $\mathrm{O}-\mathrm{H}$ bonds followed by Grotthus-like proton transfers, reorganization of hydrogen bonds preventing recombination, and modifications of $\mathrm{O}-\mathrm{O}$ and hypercoordination of $\mathrm{OH}$ groups located at strategic positions. ${ }^{1-6}$ Given the level of complexity observed in homogeneous bulk systems, it is not surprising that the above mentioned steps may show nontrivial modifications when analyzed in the presence of inhomogeneous solvating environments such as electrochemical interfaces, ${ }^{7}$ slit pores,${ }^{8}$ carbon nanotubes, ${ }^{9}$ water wires at interfaces, ${ }^{10}$ and membranes, ${ }^{11}$ to cite a few relevant examples.

The consideration of water dissociation in crystalline macroscopic phases at cryogenic conditions introduces some simplifications in the dissociation description since the absence of spatial and orientational disorders suppresses a great deal of the aforementioned fluctuations. Instead, in this thermal regime, new effects become relevant; in particular, those derived from the inherent quantum nature of the light protons. In this context, recent computer simulations performed on ice $\mathrm{I}_{\mathrm{h}}$ at temperatures close to $100 \mathrm{~K}$, showed that the incorporation of nuclear quantum effects lead to a significant increase in autoprotolysis episodes, ${ }^{12}$ normally absent in classical simulations.

Solid-like water clusters with nanometric dimensions represent another interesting benchmark to analyze water dissociation. Perhaps the simplest question to be answered in this context concerns the minimum number of water molecules that are required to stabilize zwitterionic moieties of the type $\left[\mathrm{H}_{3} \mathrm{O}\right]^{+}[\mathrm{OH}]^{-}\left(\mathrm{H}_{2} \mathrm{O}\right)_{n}$. Several quantum calculations ${ }^{13-16}$ indicate that only $n=3$ water 
molecules, arranged in a cage-like structure, would suffice to isolate charges. These observations sparked our interest to undertake computer simulations to investigate possible microscopic ionization mechanisms that, preserving the original topology of hydrogen bonds (HB), might connect neutral with ionized cluster states. In particular, we focused attention on those involving correlated Grotthus-like proton transfer events controlled by nuclear tunneling. As such, the present study is akin to a recent series of simulation analyses that examined the role of concerted proton tunneling on isotope induced charge localization in hexagonal ice. ${ }^{17-19}$ Contrasting, here, charge separation will be controlled by the intermolecular HB connectivity pattern that couples the ion pair with the rest of the cluster constituents.

The organization of this paper is as follows: In Section II we present a brief detail of the model and the simulation procedure. Additional elements are also provided in the Supplemental Information. Section III contains the simulation results whereas the main conclusions of the work are summarized in Section IV.

\section{Model and Molecular Dynamics}

The systems under investigation consisted of isolated, solid-like water nanoclusters at temperatures close to $50 \mathrm{~K}$. In particular, we focused attention on four different water moieties: the first two correspond to the $W_{5 p}$ and the $W_{8 p}$ isomers ${ }^{20}$ of the water pentamer and octamer depicted on the left-hand side of Fig. 1. For the purposes of the discussions that will follow, it will be useful to portray the geometrical characteristics of the intermolecular connectivity of these clusters in terms of non-planar, 4-ring and 6-ring structures ${ }^{21}$ comprising $\mathrm{W}_{i}$ water molecules, connected via $\mathrm{HB}_{i}$ of the type $\mathrm{O}_{i}-\mathrm{H}_{i} \cdots \mathrm{O}_{i+1}$. Intermolecular bonding along the rings is not uniform: for example, for the particular $W_{5 p}$ case, it contains three different types of molecules (see Fig. 2.a): the singledonor-double-acceptor (SDDA) molecule $\mathrm{W}_{1}$, the double-donor-single-acceptor (DDSA) molecule $\mathrm{W}_{3}$ and two single-donor-single-acceptor (SDSA) molecules $\mathrm{W}_{2}$ and $\mathrm{W}_{4}$. As such, the remaining $\mathrm{W}_{\mathrm{B}}$ water molecule will be considered as a "solvation bath", coupled to the cyclic structure at the 
SDDA and at the DDSA molecules.

Alternatively, the global structure in the $\mathrm{W}_{5 \mathrm{p}}$ cluster can be considered as composed of three, end-to-end connected branches: the $\mathrm{O}_{1}-\mathrm{H}_{1} \cdots \mathrm{O}_{2}-\mathrm{H}_{2} \cdots \mathrm{O}_{3}$ branch (hereafter referred to as the $\mathrm{B}_{1}$ branch), the $\mathrm{O}_{3}-\mathrm{H}_{3} \cdots \mathrm{O}_{4}-\mathrm{H}_{4} \cdots \mathrm{O}_{1}$ branch (hereafter referred to as the $\mathrm{B}_{2}$ branch) and a third $\mathrm{B}_{3}$ branch, equivalent to the previous one, that includes the $\mathrm{W}_{\mathrm{B}}$ molecule. A similar description holds for the larger $\mathrm{W}_{8 \mathrm{p}}$ isomer, except that the branches contain four molecules, with two SDSA molecules at intermediate positions, while the "solvaltion bath" includes two $\mathrm{W}_{\mathrm{B}}$ molecules (see Fig. 2.b).
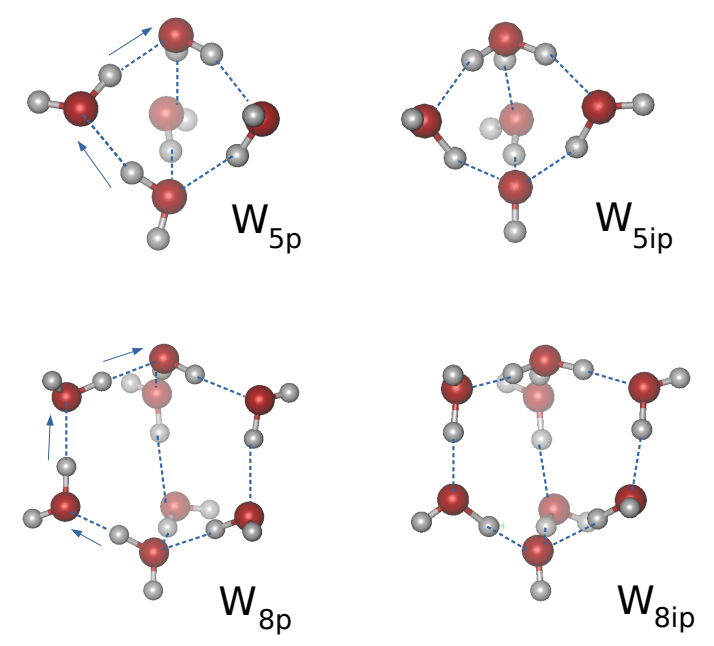

Figure 1: Snapshots of typical configurations of the $\mathrm{W}_{5 \mathrm{p}}, \mathrm{W}_{8 \mathrm{p}}, \mathrm{W}_{5 \mathrm{p} p}, \mathrm{~W}_{8 \mathrm{ip}}$ aqueous clusters. The arrows indicate collective proton motions leading to left-to-right charge separation processes (see text).

The other two water clusters that we analyzed are depicted on the right hand side of Fig. 1 and correspond to the $W_{5 i p}$ and the $W_{8 \text { ip }}$ isomers. Quantum calculations, ${ }^{13-16}$ predict that these structures represent the smallest aqueous moieties that might exhibit stable $\left[\mathrm{H}_{3} \mathrm{O}\right]^{+}[\mathrm{OH}]^{-}$ion pairs. Note that these ionic configurations can be readily obtained from those on the left-hand side via Grotthus-like, collective displacements of the protons along the corresponding $\mathrm{B}_{1}$ branches (see 
arrows in Fig. 1).
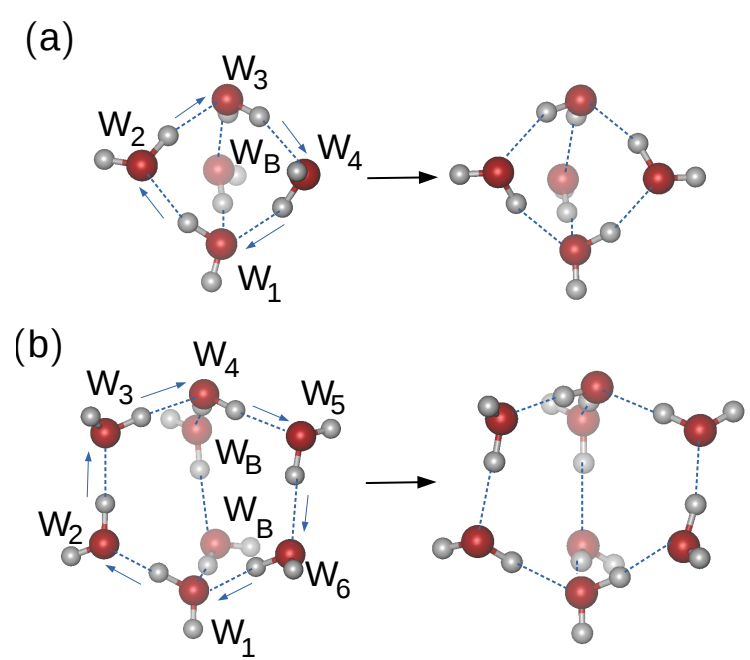

(c)

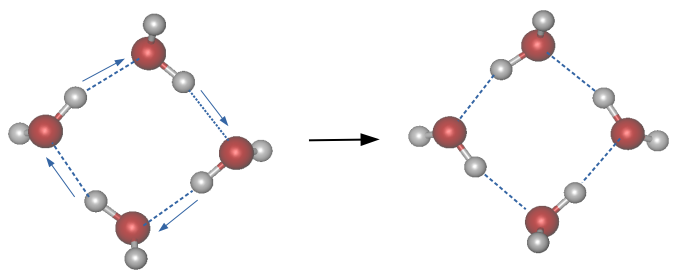

Figure 2: Reactive and product states for the exchange of donor/acceptor roles in neighboring water pairs, along the cyclic structures. (a): $\mathrm{W}_{5 \mathrm{p}}$; (b) $\mathrm{W}_{8 \mathrm{p}}$; (c) planar tetramer. The arrows indicate collective proton transfer modes leading the donor/acceptor exchanges (see text).

To perform the computer simulations we implemented a standard Path Integral Molecular Dynamics procedure. ${ }^{22}$ Briefly, the starting point of the procedure relies on the $P$-bead discretized representation of the canonical quantum partition function of an $N$ particle system with coordinates $\mathbf{r}_{N}$ at a temperature $T$, with potential energy $V:{ }^{23}$

$$
Q_{P}=\frac{1}{h^{3 P N}} \int \cdots \int \prod_{k=1}^{P} \prod_{i=1}^{N} \mathrm{~d} \mathbf{r}_{i}^{(k)} \mathrm{d} \mathbf{p}_{i}^{(k)} e^{-\beta_{P} H_{P}\left(\left\{\mathbf{p}_{i}^{(k)}\right\},\left\{\mathbf{r}_{i}^{(k)}\right\}\right)}
$$




$$
\begin{aligned}
H_{P}\left(\left\{\mathbf{p}_{i}^{(k)}\right\},\left\{\mathbf{r}_{i}^{(k)}\right\}\right)=\sum_{i=1}^{N} \sum_{k=1}^{P}\left[\frac{\left(\mathbf{p}_{i}^{(k)}\right)^{2}}{2 M_{i}}+\frac{M_{i} \omega_{P}^{2}}{2}\left(\mathbf{r}_{i}^{(k)}-\mathbf{r}_{i}^{(k+1)}\right)^{2}\right] & + \\
& +\sum_{k=1}^{P} V\left(\mathbf{r}_{1}^{(k)}, \mathbf{r}_{2}^{(k)}, \ldots, \mathbf{r}_{N}^{(k)}\right)
\end{aligned}
$$

where $P \beta_{P}=\left(k_{B} T\right)^{-1}, \omega_{P}=\left(\beta_{P} \hbar\right)^{-1}$ and $\mathbf{r}_{i}^{(k)}$ and $\mathbf{p}_{i}^{(k)}$ represent the position and momentum of the $i$-th particle of mass $M_{i}$ at the imaginary time slice $k$, respectively $\left(\mathbf{r}_{i}^{(P+1)}=\mathbf{r}_{i}^{(1)}\right)$.

Thermal averages for position dependent observables $\mathcal{O}(\{\mathbf{r}\})$ are expressed as:

$$
\langle\mathcal{O}\rangle_{P}=\frac{1}{Q_{P} h^{3 P N}} \int \cdots \int \prod_{k=1}^{P} \prod_{i=1}^{N} \mathrm{~d} \mathbf{r}_{i}^{(k)} \mathrm{d} \mathbf{p}_{i}^{(k)} e^{-\beta_{P} H_{P}} \mathcal{O}_{P}\left(\left\{\mathbf{r}_{i}^{P}\right\}\right)
$$

with

$$
\mathcal{O}_{P}\left(\left\{\mathbf{r}_{i}^{P}\right\}\right)=\frac{1}{P} \sum_{k=1}^{P} \mathcal{O}\left(\left\{\mathbf{r}_{i}^{(k)}\right\}\right)
$$

Molecular dynamics trajectories were generated by implementing a transformation from Cartesian to normal-mode coordinates, ${ }^{24}$ coupled to a multiple time step algorithm. ${ }^{25}$ The long time step was set to $0.1 \mathrm{fs}$ whereas the integration of the faster degrees of freedom involved time intervals $\delta t=\Delta t / 3$. In all cases, the clusters exhibited solid-like dynamical behavior characterized by small amplitude vibrations; no evaporation episodes were registered. The number of beads was set to $P=64$, which is similar to the discretization implemented in previous studies. ${ }^{18,26}$ A couple of examples illustrating $P$ convergence tests are presented in the Supplementary Material.

To evaluate thermodynamic information, we carried out canonical runs generated by coupling each component of each normal mode to a chain of three Nosé-Hoover thermostats ${ }^{27}$ set at $T=50$ $\mathrm{K}$. The masses of thermostats coupled to the $n$th normal mode of the polymers were set to $Q_{n}=$ $1 / \beta_{P} \omega_{n}^{2}$, where $\omega_{n}$ represents the corresponding normal mode frequency. ${ }^{28}$ In doing so, we could obtain temperature control within $0.1 \mathrm{~K}$.

Our choice for the potential energy term in Eq. (3) corresponded to the OSS2 model developed by Ojamäe et al. ${ }^{30}$ This fully atom-atom potential provides reasonable estimates for energies 
Table 1: Geometrical parameters and binding energies for clusters

\begin{tabular}{|c|c|c|c|c|c|c|c|}
\hline & OSS2 & DFT & PIMD $^{d}$ & & OSS2 & DFT & PIMD $^{d}$ \\
\hline \multicolumn{8}{|c|}{ a. ring $\left[\mathrm{H}_{2} \mathrm{O}\right]_{4}$} \\
\hline $\mathrm{E}_{d i s}$ & 126.0 & $135.7^{a}$ & & & & & \\
\hline $\mathrm{d}_{\mathrm{O}_{\mathrm{i}} \mathrm{O}_{\mathrm{i}+1}}$ & 2.83 & $2.71^{a}$ & 2.76 & $\mathrm{~d}_{\mathrm{O}_{\mathrm{i}} \mathrm{H}_{\mathrm{i}}}$ & 1.01 & $0.98^{a}$ & 1.01 \\
\hline \multicolumn{8}{|l|}{ b. $W_{5 \mathrm{p}}$} \\
\hline $\mathrm{E}_{d i s}$ & 143.4 & $137-181^{b}$ & & & & & \\
\hline $\mathrm{d}_{\mathrm{O}_{1} \mathrm{O}_{2}}$ & 2.78 & 2.66 & 2.80 & $\mathrm{~d}_{\mathrm{O}_{1} \mathrm{H}_{1}}$ & 0.99 & 1.02 & 1.02 \\
\hline $\mathrm{d}_{\mathrm{O}_{2} \mathrm{O}_{3}}$ & 2.84 & 2.69 & 2.86 & $\mathrm{~d}_{\mathrm{O}_{2} \mathrm{H}_{2}}$ & 0.99 & 1.02 & 1.02 \\
\hline $\mathrm{d}_{\mathrm{O}_{3} \mathrm{O}_{4}}$ & 2.98 & 2.86 & 2.97 & $\mathrm{~d}_{\mathrm{O}_{3} \mathrm{H}_{3}}$ & 0.98 & 0.98 & 0.99 \\
\hline $\mathrm{d}_{\mathrm{O}_{4} \mathrm{O}_{1}}$ & 2.96 & 2.91 & 2.96 & $\mathrm{~d}_{\mathrm{O}_{1} \mathrm{H}_{3}}$ & 0.97 & 0.96 & 0.99 \\
\hline \multicolumn{8}{|l|}{ c. $W_{5 i p}$} \\
\hline $\mathrm{E}_{d i s}$ & 98.6 & $85-113^{b}$ & & & & & \\
\hline $\mathrm{d}_{\mathrm{O}_{1} \mathrm{O}_{2}}$ & 2.67 & 2.52 & 2.67 & $\mathrm{~d}_{\mathrm{O}_{1} \mathrm{H}_{1}}$ & 1.67 & 1.53 & 1.67 \\
\hline $\mathrm{d}_{\mathrm{O}_{2} \mathrm{O}_{3}}$ & 2.70 & 2.52 & 2.70 & $\mathrm{~d}_{\mathrm{O}_{2} \mathrm{H}_{2}}$ & 1.73 & 1.51 & 1.75 \\
\hline \multicolumn{8}{|l|}{ d. $W_{8 p}$} \\
\hline $\mathrm{d}_{\mathrm{O}_{1} \mathrm{O}_{2}}$ & 2.77 & $2.62^{c}$ & 2.79 & $\mathrm{~d}_{\mathrm{O}_{1} \mathrm{H}_{1}}$ & 1.03 & $1.00^{c}$ & 1.03 \\
\hline $\mathrm{d}_{\mathrm{O}_{2} \mathrm{O}_{3}}$ & 2.76 & $2.63^{c}$ & 2.78 & $\mathrm{~d}_{\mathrm{O}_{2} \mathrm{H}_{2}}$ & 1.02 & $1.00^{c}$ & 1.02 \\
\hline $\mathrm{d}_{\mathrm{O}_{3} \mathrm{O}_{4}}$ & 2.80 & $2.63^{c}$ & 2.81 & $\mathrm{~d}_{\mathrm{O}_{3} \mathrm{H}_{3}}$ & 1.03 & $1.00^{c}$ & 1.02 \\
\hline $\mathrm{d}_{\mathrm{O}_{4} \mathrm{O}_{5}}$ & 2.93 & $2.80^{c}$ & 2.96 & $\mathrm{~d}_{\mathrm{O}_{4} \mathrm{H}_{4}}$ & 1.00 & $0.98^{c}$ & 0.99 \\
\hline $\mathrm{d}_{\mathrm{O}_{5} \mathrm{O}_{6}}$ & 2.88 & $2.80^{c}$ & 2.90 & $\mathrm{~d}_{\mathrm{O}_{5} \mathrm{H}_{5}}$ & 1.00 & $0.98^{c}$ & 0.99 \\
\hline $\mathrm{d}_{\mathrm{O}_{6} \mathrm{O}_{1}}$ & 2.94 & $2.74^{c}$ & 2.87 & $\mathrm{~d}_{\mathrm{O}_{5} \mathrm{H}_{6}}$ & 1.00 & $0.98^{c}$ & 0.99 \\
\hline \multicolumn{8}{|l|}{ e. W8ip } \\
\hline $\mathrm{E}_{d i s}$ & 225.4 & $183-276^{b}$ & & & & & \\
\hline $\mathrm{d}_{\mathrm{O}_{1} \mathrm{O}_{2}}$ & 2.71 & $2.54^{c}$ & 2.71 & $\mathrm{~d}_{\mathrm{O}_{1} \mathrm{H}_{1}}$ & 1.67 & $1.51^{c}$ & 1.65 \\
\hline $\mathrm{d}_{\mathrm{O}_{2} \mathrm{O}_{3}}$ & 2.68 & $2.57^{c}$ & 2.71 & $\mathrm{~d}_{\mathrm{O}_{2} \mathrm{H}_{2}}$ & 1.65 & $1.55^{c}$ & 1.68 \\
\hline $\mathrm{d}_{\mathrm{O}_{3} \mathrm{O}_{4}}$ & 2.70 & $2.52^{c}$ & 2.69 & $\mathrm{~d}_{\mathrm{O}_{3} \mathrm{H}_{3}}$ & 1.70 & $1.49^{c}$ & 1.69 \\
\hline
\end{tabular}

${ }^{b}$ Results reported in Ref. [29] using different levels of approximation within the B3LYP functional scheme. ${ }^{c}$ The distances correspond to B3LYP-D3 results from Ref. [29]. $\quad{ }^{d}$ PIMD statistical averages evaluated at $50 \mathrm{~K}$. Energy values are expressed in $\mathrm{kJ} \mathrm{mol}^{-1}$ and lengths are expressed in $\AA$.

and geometrical parameters of excess protons in water clusters and neutral aggregates as well. In addition, it incorporates dissociative pathways and can be implemented within the PIMD scheme in a straightforward fashion, allowing appropriate harvesting of statistics at reasonable computing costs. As a direct test to gauge the accuracy of the predictions from the OSS2 Hamiltonian, in Table I we list results for a series of relevant interatomic distances and binding energies for quenched 
structures (obtained via simulated annealing) along with those obtained from representative B3LYP density functional theory estimates recently reported in the literature. ${ }^{29}$ In all cases, OSS2 binding energies fall within the dispersion obtained by considering different levels of approximation. On the other hand, differences between the OSS2 results for the interatomic distances and DFT predictions remain within $5-10 \%$.

\section{Results}

\subsection{Collective proton transfer along cyclic structures}

Before addressing the analysis of the mechanisms that drive charge separation in water clusters, it will be instructive to examine the related proton transfer isomerizations involving the modes depicted in Fig. 2. These modes are characterized by collective displacements of all the protons along the ring structures; the net result of these displacements is the exchange in the HB acceptor/donor roles in nearest neighbor water pairs, namely:

$$
\mathrm{O}_{\mathrm{i}}-\mathrm{H}_{\mathrm{i}} \cdots \mathrm{O}_{\mathrm{i}+1} \rightarrow \mathrm{O}_{\mathrm{i}} \cdots \mathrm{H}_{\mathrm{i}}-\mathrm{O}_{\mathrm{i}+1}
$$

Following previous analysis, ${ }^{17,18,26}$ an adequate collective variable to describe such exchange of roles is given by the equally weighted sum of asymmetric stretching coordinates:

$$
\bar{\xi}=\frac{1}{N_{c}} \sum_{i=1}^{N_{c}} \xi_{i}
$$

where

$$
\xi_{i}=\left|\mathbf{r}_{\mathrm{O}}^{i}-\mathbf{r}_{\mathrm{H}}^{i}\right|-\left|\mathbf{r}_{\mathrm{O}}^{i+1}-\mathbf{r}_{\mathrm{H}}^{i}\right|
$$

In Eq. $6, N_{c}$ corresponds to the number of water molecules comprising the cycle $\left(\mathbf{r}_{\mathrm{O}}^{N_{c}+1}=\mathbf{r}_{\mathrm{O}}^{1}\right)$. Within the path integral context, the latter definition can be naturally extended to an equivalent 
expression in terms of distances between atomic centroids $\left\{\mathbf{r}_{N}^{\mathrm{cnt}}\right\},{ }^{17,18,26,31}$ where:

$$
\mathbf{r}_{i}^{\mathrm{cnt}}=\frac{1}{P} \sum_{k=1}^{P} \mathbf{r}_{i}^{(k)}
$$

Armed with these definitions, we explored the characteristics of the reactive path along the $\bar{\xi}$ coordinate by constructing the corresponding free energy profile $A\left(\xi^{\prime}\right)$ :

$$
\beta A\left(\xi^{\prime}\right) \propto-\ln \left\langle\delta\left(\bar{\xi}\left(\left\{\mathbf{r}_{N}^{\text {cnt }}\right\}\right)-\xi^{\prime}\right)\right\rangle
$$

where $\langle\cdots\rangle$ denote an equilibrium ensemble average. We remark that, given the non-linear nature of the collective variable, the previous equation represents an approximate expression to the more rigorous treatment that, according to Eq. (5), would involve the following "bead average" computation:

$$
\beta A\left(\xi^{\prime}\right) \propto-\ln \left[\frac{1}{P} \sum_{k=1}^{P}\left\langle\delta\left(\bar{\xi}\left(\left\{\mathbf{r}_{N}^{(k)}\right\}\right)-\xi^{\prime}\right)\right\rangle\right]
$$

The exchange reaction involves ruptures and reconstructions of intramolecular $\mathrm{O}-\mathrm{H}$ bonds; in the gas phase, the energy cost of such processes is typically of the order of hundreds of kcal $\mathrm{mol}^{-1}$. Consequently, proper sampling of all relevant regions of phase space will necessarily require the incorporation of a non-Boltzmann sampling strategy. In this case, we found that the direct implementation of standard umbrella sampling techniques ${ }^{32}$ turned out to be adequate to collect statistics along the complete $\xi$ interval of interest (see the Supplemental Information for further details of our implementation).

Results for the PIMD $A(\xi)$ for the pentamer are presented in Fig. 3 (red squares). The profile is symmetric with respect to the transition state located a $\xi=0$ and exhibits two distinct regimes: the first one, hereafter denoted $\mathcal{A}$, is characterized by a parabolic-like shape, and spans along the $\xi<-0.6 \AA$ interval. A sharp transition into a $\mathcal{B}$ plateau regime takes place as one surpasses the latter value; beyond this threshold, the free energy difference levels off at $\sim 6.1 \mathrm{kcal} \mathrm{mol}^{-1}$, reaching the transition state practically without any further modifications. 
Figure 3: Free energy profiles associated with the donor-acceptor exchange along cyclic structures in water clusters. $\left[\mathrm{H}_{2} \mathrm{O}\right]_{5}$, PIMD: red squares; $\left[\mathrm{H}_{2} \mathrm{O}\right]_{4}$, PIMD: black circles; $\left[\mathrm{H}_{2} \mathrm{O}\right]_{4}$, classic: solid lines; $\left[\mathrm{H}_{2} \mathrm{O}\right]_{8}$, PIMD: violet triangles. The dashed vertical lines represent approximate boundaries between regimes $\mathcal{A}$ and $\mathcal{B}$ (see text). For clarity purposes, only the $\xi<0$ portions of the symmetric plots are shown.

The direct comparison between these features with those perceived from the plot with black circles corresponding to the ring $\left[\mathrm{H}_{2} \mathrm{O}\right]_{4}$ (see Fig. 2.c) is instructive. Note that both curves exhibit $\mathcal{A}$-to- $\mathcal{B}$ transitions located at similar values of $\xi$; however, the absence of coupling with the $\mathrm{W}_{\mathrm{B}}$ molecule, lowers the magnitude of the activation energy in the water tetramer by approximately 1 $\mathrm{kcal} \mathrm{mol}^{-1}$. Moreover, the previous characteristics of the PIMD results contrast sharply to those obtained from a similar calculation performed for the classical, i.e. the $P=1$ case, water tetramer, in which nuclear quantum effects are switched off. In the latter case, the free energy plot (solid black line) exhibits the usual double well shape, reflecting the characteristics of the potential energy surface, with reactant and product states separated by a five-fold higher activation energy barrier (see inset). These observations reveal modifications not only at quantitative but also at qualitative levels that deserve further investigation. In passing, we remark that similar characteristics have also been reported for collective proton transfer processes in hexagonal ice rings at low temperatures ${ }^{26}$ and diffusion of Hydrogen molecules in crystalline solids. ${ }^{31}$

To move forward in our analysis, we examined the average values of the geometrical parameters related to the set of the individual $\xi_{i} \mathrm{~s}$. These average values were obtained from restricted samplings, using umbrella potentials, centered at different values $\bar{\xi}$ of interest and will be denoted 


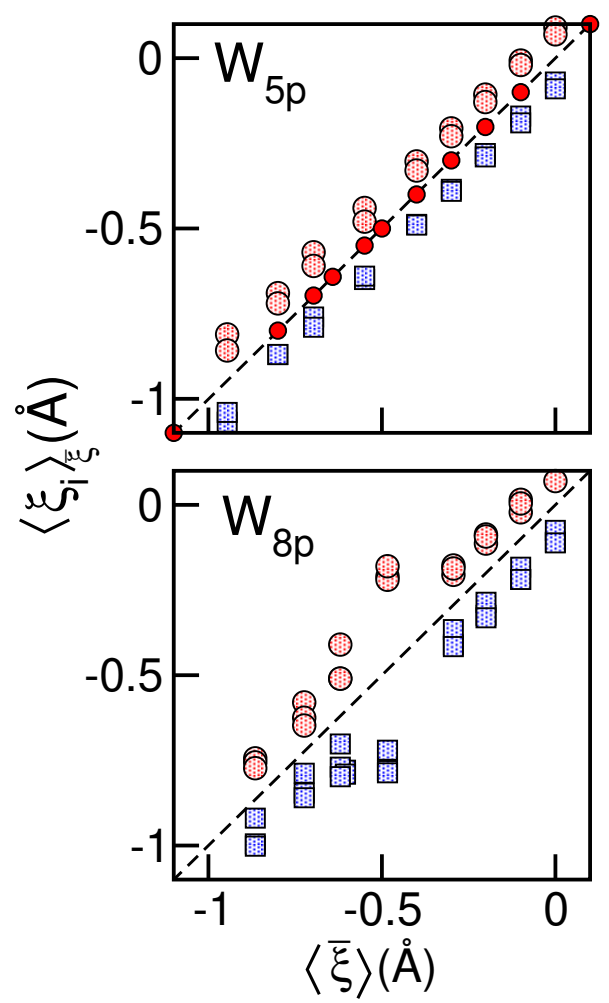

Figure 4: Statistical averages of stretching coordinates $\xi_{i}$ along collective cyclic modes in the $\mathrm{W}_{5 \mathrm{p}}$ isomer (top panel) and in the $\mathrm{W}_{8 \mathrm{p}}$ isomer (bottom panel). Results for the $\mathrm{B}_{1}$ branch (dotted red circles); results for the $\mathrm{B}_{2}$ branch (dotted blue squares). The solid red circles correspond to results for the planar tetramer.

as $\langle\cdots\rangle_{\bar{\xi}}$. The top panel of Fig. 4 contains results for $\left\langle\xi_{i}\right\rangle_{\bar{\xi}}$ for the planar tetramer and the pentamer. Interestingly, the two sets of average values corresponding to the $\mathrm{B}_{1}$ branch were consistently $\sim 0.2$ $\AA$ higher than those corresponding to the $\mathrm{B}_{2}$ branch. This feature would indicate that "solvation effects" induced by the coupling between the ring and the $\mathrm{W}_{B}$ molecule lead to a mild tendency to localize positive and negative charges at the positions of the $\mathrm{W}_{1}$ and $\mathrm{W}_{3}$ molecules. Of course these tendencies are absent in the isolated water tetramer, where the four $\left\langle\xi_{i}\right\rangle_{\bar{\xi}}$ coincide within the statistical error (see red circles), revealing practically perfect correlation between the individual transfers. Still, these solvation effects do not provide sufficient clues to rationalize neither the similarities nor the differences between the pentamer and the tetramer free energy plots.

A further decomposition of the individual values of $\xi_{i}$ into distances between donor-acceptor 
Figure 5: Panels (a) and (b): Statistical averages of $\mathrm{O}_{\mathrm{i}}-\mathrm{H}_{\mathrm{i}}$ and $\mathrm{O}_{\mathrm{i}}-\mathrm{O}_{\mathrm{i}+1}$ distances at different $\mathrm{HBs}$ along the collective cyclic mode in the $\mathrm{W}_{5 \mathrm{p}}$ cluster. $\mathrm{HB}_{2}$ : open red triangles; $\mathrm{HB}_{3}$ : solid red triangles. Panels (c) and (d): similar results for the $\mathrm{W}_{8 \mathrm{p}}$ cluster. $\mathrm{HB}_{3}$ : open violet squares; $\mathrm{HB}_{4}$ : solid violet squares. The vertical dashed lines indicate $\mathcal{A}$-to- $\mathcal{B}$ regime transitions (see text). The open black circles in panels (a) and (b) correspond to results for the planar tetramer.

oxygens $\left(d_{\mathrm{O}_{\mathrm{i}} \mathrm{O}_{\mathrm{i}+1}}\right)$ and donor oxygen-hydrogen pairs $\left(d_{\mathrm{O}_{\mathrm{i}} \mathrm{H}_{\mathrm{i}}}\right)$ proved to be more enlightening. As representative examples, in panels (a) and (b) of Fig. 5 we present results for averages of these distances for two $\mathrm{H}$-bonds located in different branches: $\mathrm{HB}_{2}$ and $\mathrm{HB}_{3}$. In the two sets of plots, the sharp changes in the tendencies, already observed in the free energy profiles at the $\mathcal{A}$-to- $\mathcal{B}$ transitions, are also reproduced. In particular, along the $\mathcal{A}$ stage, the average values of $d_{\mathrm{O}_{\mathrm{i}} \mathrm{H}_{\mathrm{i}}}$ exhibit only minor changes; as a result, the increase of $\langle\bar{\xi}\rangle$ is practically the result of the gradual contraction of $d_{\mathrm{O}_{\mathrm{i}} \mathrm{O}_{\mathrm{i}+1}}$ from $2.9 \AA(2.8 \AA)$ down to $2.7 \AA(2.6 \AA)$ for $i=2(i=3)$. The transition observed at $\langle\bar{\xi}\rangle \sim-0.6$ coincides with sharp relaxations of the contracted $d_{\mathrm{O}_{\mathrm{i}} \mathrm{O}_{\mathrm{i}+1}}$, concomitant with the onset of steady stretchings of the different $d_{\mathrm{O}_{\mathrm{i}} \mathrm{H}_{\mathrm{i}}}$, which now represent the key elements controlling the overall changes in $\langle\bar{\xi}\rangle$. Note that these behaviors are also observed in the results for the ring $\left[\mathrm{H}_{2} \mathrm{O}\right]_{4}$ cluster, also depicted in the Figure with open black circles.

A last element is still needed to complete the previous description and deals with the extent of the spatial localization of the transferred quantum protons along the different stages of the reactive path. To gauge the magnitude of these changes, we computed two different kinds of 
spatial correlations. The first one involves the analysis of bead-densities projected along $\mathrm{O}_{i}-\mathrm{O}_{i+1}$ directions, at different values of the umbrella potential, namely:

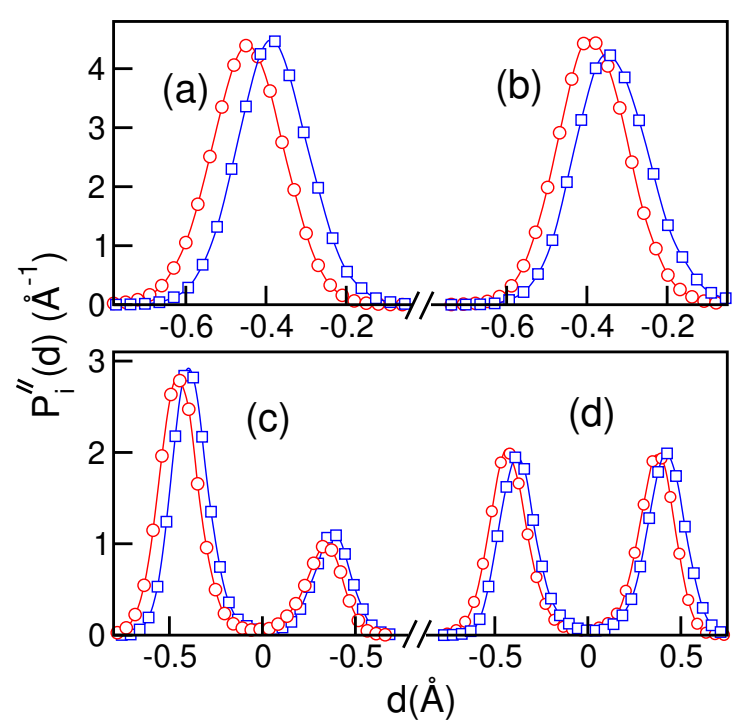

Figure 6: Spatial bead-density projected along the $\mathrm{O}_{i}-\mathrm{O}_{i+1}$ direction for $i=2$ (open blue squares) and $i=3$ (open red circles) at four different values of $\langle\bar{\xi}\rangle$. Top panel: (a): $\langle\bar{\xi}\rangle=-0.86 \AA$; (b): $\langle\bar{\xi}\rangle=-0.6 \AA$. Bottom panel: $(\mathrm{c}):\langle\bar{\xi}\rangle=-0.3 \AA ;(\mathrm{d}):\langle\bar{\xi}\rangle=0.0 \AA$.

$$
P_{i}^{\|}(d)=\frac{1}{P} \sum_{k=1}^{P}\left\langle\delta\left(d_{i}^{(k)}-d\right)\right\rangle_{\bar{\xi}}
$$

where

$$
d_{i}^{(k)}=\left(\mathbf{r}_{\mathrm{H}_{\mathrm{i}}}^{(k)}-\overline{\mathbf{r}}_{\mathrm{O}_{\mathrm{i}} \mathrm{O}_{\mathrm{i}+1}}\right) \cdot \hat{\mathbf{u}}_{\mathrm{O}_{\mathrm{i}} \mathrm{O}_{\mathrm{i}+1}}
$$

with

$$
\overline{\mathbf{r}}_{\mathrm{O}_{\mathrm{i}} \mathrm{O}_{\mathrm{i}+1}}=\frac{\mathbf{r}_{\mathrm{O}_{\mathrm{i}}}^{\mathrm{cnt}}+\mathbf{r}_{\mathrm{O}_{\mathrm{i}+1}}^{\mathrm{cnt}}}{2}
$$

and

$$
\hat{\mathbf{u}}_{\mathrm{O}_{\mathrm{i}} \mathrm{O}_{\mathrm{i}+1}}=\frac{\mathbf{r}_{\mathrm{O}_{\mathrm{i}+1}}^{\mathrm{cnt}}-\mathbf{r}_{\mathrm{O}_{\mathrm{i}}}^{\mathrm{cnt}}}{\left|\mathbf{r}_{\mathrm{O}_{\mathrm{i}+1}}^{\mathrm{cnt}}-\mathbf{r}_{\mathrm{O}_{\mathrm{i}}}^{\mathrm{cnt}}\right|}
$$

Results for $P_{i}^{\|}(d)(i=2,3)$ computed at four representative values of $\langle\bar{\xi}\rangle$, appear in Fig. 6. As a common feature, all $P_{2}^{\|}$distributions appear shifted by $\sim 0.1 \AA$ with respect to those corresponding 
to the $P_{3}^{\|}$, reflecting the already mentioned differences in the extent of charge transfer along the two branches of the ring. More noticeable are the changes operated in the overall shapes of the plots as one compares scenarios $\mathcal{A}$ and $\mathcal{B}$. The plots in the upper panel correspond to domains $\mathcal{A}$ and look fairly Gaussian-like, with widths of $\sim 0.2 \AA$. This reveals that the proton isomorphic polymers present compact, coiled structures, located at approximately $\sim 1 \AA$ from the donor oxygens.

Contrasting, along the $\mathcal{B}$ realm (see plots (c) and (d) in the bottom panel), nuclear tunneling prevails, giving rise a picture characterized by stretched polymers localized at the two minima of the bistable local potential energy profile, with fleeting imaginary time interwell transitions. According to this picture, the profiles indicate that the progress in the proton transfers towards the transition state is operated by a steady migration of larger fractions of polymer beads from the donor basin of attraction located at $d=-0.45 \AA$, towards the acceptor basin located a $d=0.45 \AA$.

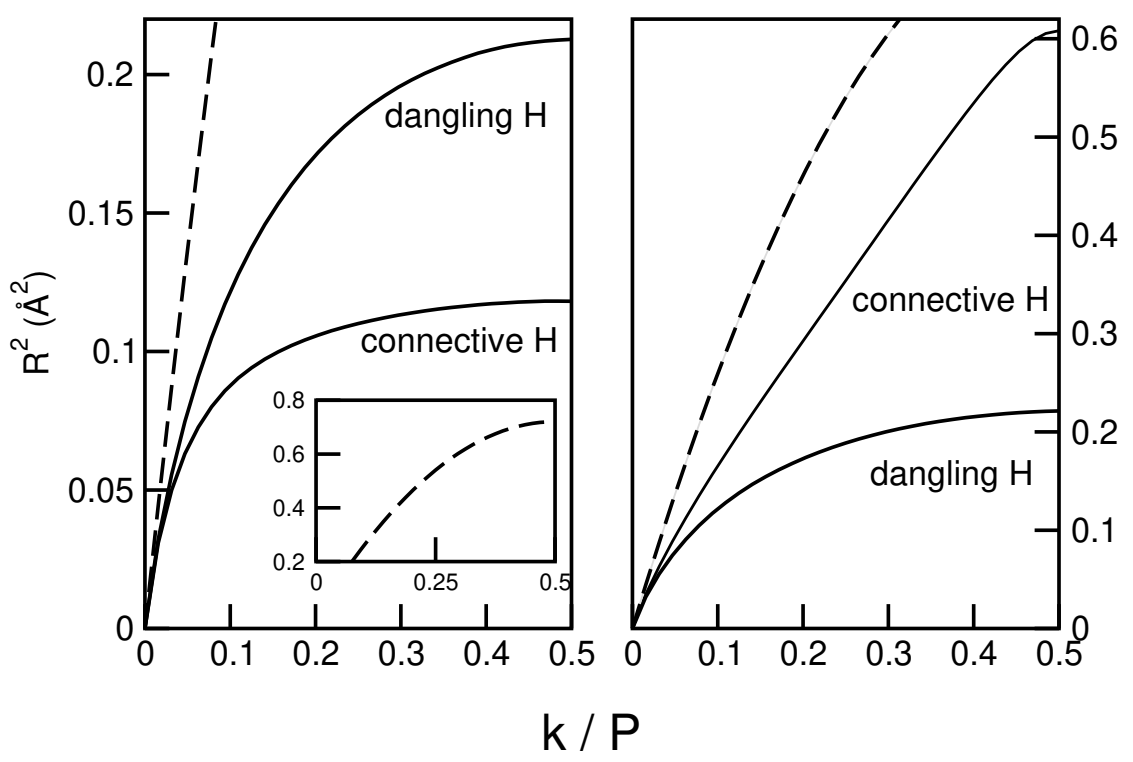

Figure 7: Imaginary time mean square displacements for the different hydrogens in the cyclic tetramer at $T=50 \mathrm{~K}$. Left panel: sampling at reactant/product states; $\bar{\xi} \sim \pm 0.84 \mathrm{~A}$. Right panel: sampling at the transition state; $\bar{\xi}=0 \AA$. Also shown are results for thermalized, non interacting protons, $\mathcal{R}_{\text {free }}^{2}(k)$ (dashed lines)

The second correlations that we analyzed involve intrapolymer spatial distributions; more 
specifically we examined proton correlation lengths, $\mathcal{R}$, computed from imaginary time mean square displacements of the type: ${ }^{33}$

$$
\mathcal{R}_{i}^{2}(k)=\frac{1}{P} \sum_{j=1}^{P}\left\langle\left|\mathbf{r}_{\mathrm{H}_{\mathrm{i}}}^{(j+k)}-\mathbf{r}_{\mathrm{H}_{\mathrm{i}}}^{(j)}\right|^{2}\right\rangle_{\bar{\xi}} \quad ; 0 \leq k \leq P .
$$

In the last equation, $\mathbf{r}_{\mathrm{H}_{\mathrm{i}}}$ denotes the position of the a tagged $\mathrm{H}$-site along the cycle (i=connective, dangling). The correlation lengths correspond to the maximum values of the mean square displacements, $\mathcal{R}_{i}=\mathcal{R}_{i}(P / 2)$. For thermalized, free (i.e. non interacting) protons, $\mathcal{R}_{\text {free }}=0.85 \AA .{ }^{33}$ The left panel in Fig. 7 contains plots for $\mathcal{R}_{i}^{2}(k)$ for reactant and product states of the illustrative case of the cyclic tetramer. The results do not differ substantially from those reported for the water octamer at similar temperatures (see Fig. 3 of Ref. [34] ). Intramolecular interactions bring the value of $\mathcal{R}_{\mathrm{H}_{\mathrm{dng}}}$ down to $\sim 0.5 \mathcal{R}_{\text {free }}$, whereas intermolecular interactions along HBs promote an additional $\sim 0.1 \AA$ contraction in the resulting value of $\mathcal{R}_{\mathrm{H}_{\mathrm{con}}}$. Modifications in the spatial extents operated by nuclear tunneling are much more transparent at the transition state: The results in the right-hand side panel show that, while the $\mathcal{R}_{\mathrm{H}_{\mathrm{dng}}}^{2}$ plot presents no meaningful modifications, the curve of $\mathcal{R}_{\mathrm{H}_{\text {con }}}^{2}$ is characterized by a much larger variety of lengthscales. In this case, the resulting correlation length stretches out up to $\sim 0.8 \AA$, a value comparable to the above mentioned distance separating donor/acceptor basins of attraction.

The picture that emerges from the previous considerations can be summarized as follows: $(i)$ the magnitude of the PIMD free energy barrier along the collective $\bar{\xi}$ reactive path is mainly determined by the energy cost required to approach neighboring oxygen atoms from typical distances of reactant states, say 2.8-2.95 $\AA$, down to values intermediate between 2.6 and $2.7 \AA$; $(i i)$ during the $\mathcal{A}$ stage, the distances between the protons and the oxygen atoms in the donor molecules remain practically unchanged so the modifications in $\bar{\xi}$ go hand-in-hand with those registered in the $\mathrm{O}_{i}-\mathrm{O}_{i+1}$ distances; $(i i i)$ : the PIMD averages for the latter distances in the $\mathrm{W}_{5 \mathrm{p}}$ isomer at the reactive state are $\sim 0.15-0.2 \AA$ longer than in the cyclic tetramer (see entries in column 4 of Table I). A simple calculation shows that such modifications are translated in a $\sim-0.12 \AA$ shift in 
the locations of the minima of the corresponding free energy curves observed in Fig. 3 and would explain the $1 \mathrm{kcal} \mathrm{mol}^{-1}$ increment in the reversible work which is necessary to bring the nuclei to the $\mathcal{A}$-to- $\mathcal{B}$ transition; $(i v)$ the compressions of the $\mathrm{O}_{i}-\mathrm{O}_{i+1}$ below a threshold distance modulates the magnitude of the barrier controlling nuclear tunneling which is operated by the gradual migration of the proton beads from one minimum of the local bistable potential to the adjacent one; $(v)$ given the still much higher magnitudes of the intrinsic potential energy barriers that persist along the different $\mathrm{O}_{\mathrm{i}}-\mathrm{O}_{\mathrm{i}+1}$ ring edges compared to typical thermal energies, the fraction of bead populations located away from the attractive wells remain negligible; $(v i)$ as such, the extra energy cost for bead migration gets drastically reduced along the $\mathcal{B}$ regime which, in turn, is reflected in the observed quasi-plateau behavior. Note that the possibility of gradual charge transfer operated by inter-well transitions at practically no energy costs contrasts sharply with the classical, $P=1$ situation, which would otherwise require surmounting the local potential energy barriers and explains the differences between the quantum and classical activation energies shown in Fig. 3.

We will close this section by commenting on the characteristics of collective transfers along the six water ring of the $\mathrm{W}_{8 \mathrm{p}}$ cluster. The corresponding free energy profile (shown with open violet triangles in Fig. 3) shows features similar to the ones already described for the $\mathrm{W}_{5 \mathrm{p}}$ cluster, except that the positions of the minimum and of the $\mathcal{A}$-to- $\mathcal{B}$ transition are shifted by $0.1 \AA$ and $0.14 \AA$, respectively. As a result, the free energy plateau rises an additional $\sim 2.2 \mathrm{kcal} \mathrm{mol}^{-1}$. The comparison between the plots in the two panels composing Fig. 4 reveals differences between the degree of correlation between proton transfers along the two different branches. The loss of correlation is clearly visible in the bottom panel at $\langle\bar{\xi}\rangle \sim-0.5 \AA$, and is also evident in the behaviors of the interatomic distances, most notably in the $\mathrm{O}_{\mathrm{i}}-\mathrm{O}_{\mathrm{i}+1}$ distances (see lower panel on the right-hand side of Fig. 5). In particular, compressions in the $\mathrm{O}_{\mathrm{i}}-\mathrm{O}_{\mathrm{i}+1}$ distances along the $\mathrm{B}_{1}$ branch are more marked, facilitating the "earlier" detachment of the protons from the donor molecules operated via tunneling. A clear picture of the latter feature is illustrated in the results shown in Fig. 8 that correspond to imaginary time stretching coordinates, ${ }^{26}$ namely: 


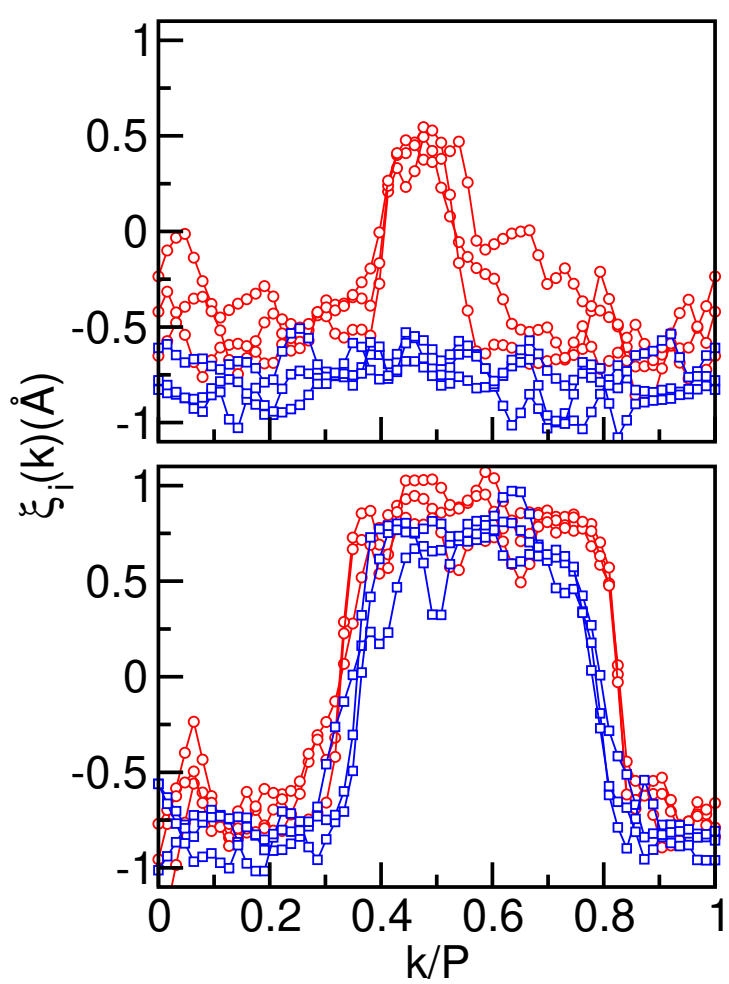

Figure 8: Imaginary time asymmetric stretching coordinates for the three protons in the $\mathrm{B}_{1}$ branch (red circles) and in the $\mathrm{B}_{2}$ branch (blue squares) of the $\mathrm{W}_{8 \mathrm{p}}$ cluster. The results correspond to two representative configurations for runs in which $\langle\bar{\xi}\rangle=-0.55 \AA$ (top panel) and $\langle\bar{\xi}\rangle=0.0 \AA$ (bottom panel).

$$
\xi_{i}(k)=\left|\mathbf{r}_{\mathrm{O}_{\mathrm{i}}}^{(k)}-\mathbf{r}_{\mathrm{H}_{\mathrm{i}}}^{(k)}\right|-\left|\mathbf{r}_{\mathrm{O}_{\mathrm{i}+1}}^{(k)}-\mathbf{r}_{\mathrm{H}_{\mathrm{i}}}^{(k)}\right| \quad ; 0 \leq k \leq P
$$

for two representative configurations corresponding to a partial tunneling regime, $\langle\bar{\xi}\rangle=-0.55$ $\AA$ (top panel), and to the transition state, $\langle\bar{\xi}\rangle=0.0 \AA$ (bottom panel). The differences in the two sets of plots are selfevident: in the former case, $\sim 20 \%$ of the hydrogen beads along the $\mathrm{B}_{1}$ branch have already been transferred from the reactant to product states. Contrasting, along the $\mathrm{B}_{2}$ branch, the protons remain localized at the reactant basins as the contractions in the distances between donor/acceptor pairs seem to be still insufficient to promote collective proton tunneling. This scenario presents some elements reminiscent of the disruption of collective proton tunneling induced by partial deuteration in the cyclic water hexamer, ${ }^{17}$ although here, such inhibition seems 
to be controlled by the characteristics of the local spatial asymmetries of the Coulomb coupling between the reactive ring and the rest of the cluster constituents. The results at the transition state appear in the bottom panel and differ at a qualitative level; at a first glance, the plots reveal practically perfect correlation between the six proton transfer steps at each imaginary time slice. Yet, a closer examination reveals that the values of $\xi_{i}^{(k)}$ for the $\mathrm{B}_{1}$ branch (red open circles) are consistently $\sim 0.1 \AA$ higher than those corresponding to the $\mathrm{B}_{2}$ branch (blue open squares), reflecting the global polarization along the $\mathrm{O}_{1}-\mathrm{O}_{4}$ axis, induced by the pair of $\mathrm{W}_{\mathrm{B}}$ molecules.

\subsection{Charge separation via collective proton transfer}

The previous analysis can be extended to the examination of $\left[\mathrm{H}_{3} \mathrm{O}\right]^{+}-[\mathrm{OH}]^{-}$charge separation processes in a straightforward fashion by simply restricting the reactive moiety to the $\mathrm{B}_{1}$ branch while the bath will include the rest of the cluster constituents. Since many of the considerations already reported remain valid for the present case, for the sake of concision, we will only comment on those exhibiting meaningful differences.

In Fig. 9 we present results for classical (solid lines) and PIMD (open circles) free energy profiles. To facilitate the descriptions of the classic curves, it will be useful to discriminate different regimes. The already mentioned parabolic behavior at low values of $\xi$ is characterized by the compressions of $\mathrm{O}_{\mathrm{i}}-\mathrm{O}_{\mathrm{i}+1}$ distances. The product states are associated with minima lying $\sim 10-12 \mathrm{kcal} \mathrm{mol}^{-1}$ above the reactant states and located at $\xi \sim-0.7 \AA$. The third intermediate regime corresponds to solvation-induced, $\sim 2 \mathrm{kcal} \mathrm{mol}^{-1}$ barriers (measured from the corresponding product sides) which, in principle, would be sufficiently high compared to typical thermal energies to prevent spontaneous recombinations of the ionic species at microscopic characteristic timescales. Note that, the resulting zwitterionic configurations of the $\mathrm{W}_{5 i p}$ cluster are akin to those usually referred to as "solvent-shared-ion-pairs" in solution where the two ions are separated by a single layer of three solvent molecules; similarly, configurations corresponding to the $\mathrm{W}_{8 \mathrm{ip}}$ cluster could be assimilated to "solvent-separated-ion-pairs", characterized by adjacent individual solvation shells, each one comprising three water molecules. 


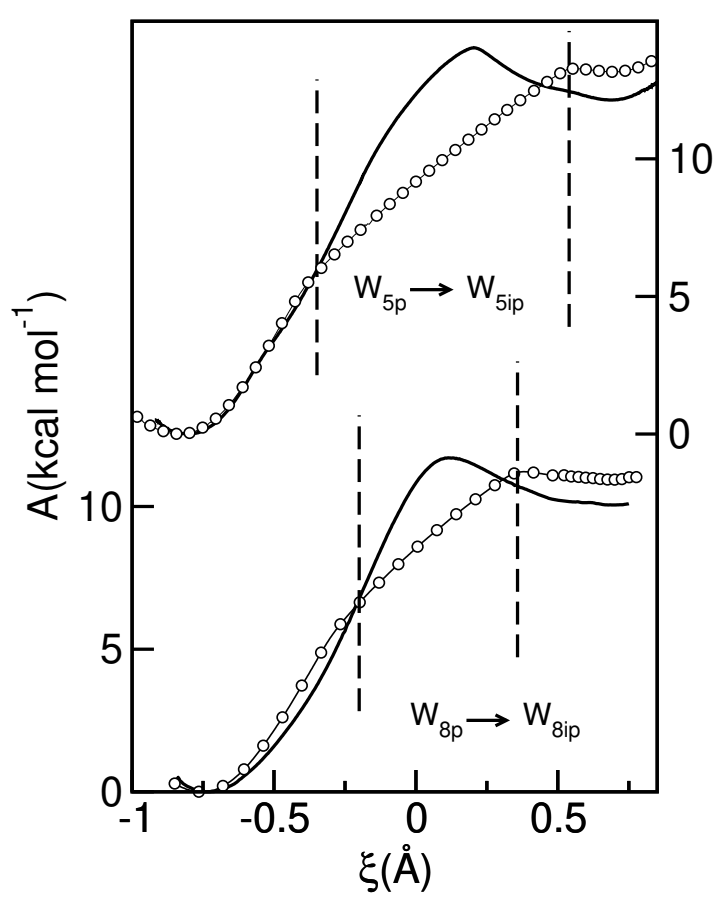

Figure 9: Free energy profiles associated with charge separation in $\left[\mathrm{H}_{2} \mathrm{O}\right]_{5}$ (right-hand $y$-axis) and $\left[\mathrm{H}_{2} \mathrm{O}\right]_{8}$ (left-hand $y$-axis). Classical results: solid lines; PIMD results: open circles. The dashed lines represent approximated boundaries separating three different regimes (see text).

The explicit incorporation of nuclear quantum effects in the simulations promotes interesting modifications in the free energy profiles: most notable ones are those registered in the latter intermediate stage, where the former stabilizing barriers get replaced by quasi-linear regimes joining reactant and product states. In addition, one also observes $\sim 1 \mathrm{kcal} \mathrm{mol}^{-1}$ increments in the product-reactant free energy differences.

This observation is particularly relevant since it indicates that the more rigorous quantum treatment would cause charge separated species to be more unstable, opening possibilities for fast recombination channels. Given the characteristics of the $\mathrm{HB}$ connectivity of the $\mathrm{W}_{5 \mathrm{ip}}$ and the $\mathrm{W}_{8 \mathrm{ip}}$ isomers, such recombinations could be funneled either retracing the original $\mathrm{B}_{1}$ branch activation paths or, alternatively, along the other two branches, with equal probabilities. Note that, should these event occur, the net result of the charge separation-recombination-tandem process would be equivalent to the isomerization reaction previously described, operated now along a different reac- 
Figure 10: Time evolutions of asymmetric stretching coordinates (top panel) and $\mathrm{O}_{i}-\mathrm{O}_{i+1}$ distances (bottom panel) along ten statistically independent charge recombination processes in the $\mathrm{W}_{\text {8ip }}$ cluster. Relaxations along the reactive branches are shown with red lines; results for the two other branches are shown with black lines.

tion path involving short-lived, charge-separated, intermediate states lying at higher free energies. In addition, note that the tunneling mechanism consisting in the gradual transfer of beads between adjacent basins of attraction of asymmetric double-well potentials would also account for the linear characteristics of the profiles at the intermediate regimes, which could be interpreted as the result of population-weighted averages of the two energy minima of the potentials.

Concerning the $\sim 1 \mathrm{kcal} \mathrm{mol}^{-1}$ increments of the quantum free energy differences between reactant and product states, we remark that these differences can be obtained via an alternative procedure involving classical and quantum computations of free energies for reactants and products. Following Ref. [35], these differences can be conveniently computed by implementing a scaled coordinate $(\mathrm{SC})$ procedure, namely:

$$
\Delta A_{\alpha}^{\mathrm{cl} \rightarrow \mathrm{qn}}=\int_{0}^{1} \mathrm{~d} \lambda A_{\alpha}^{\mathrm{SC}}(\lambda)
$$


where

$$
A_{\alpha}^{\mathrm{SC}}(\lambda)=\frac{1}{P} \sum_{i=1}^{N} \sum_{k=1}^{P}\left\langle\left(\mathbf{r}_{i}^{(k)}-\mathbf{r}_{i}^{c}\right) \frac{\partial U\left(\left\{\tilde{\mathbf{r}}_{i}^{(k)}\right\}\right)}{\partial \tilde{\mathbf{r}}_{i}^{(k)}}\right\rangle_{\lambda, \alpha}
$$

In the previous equation $\langle\cdots\rangle_{\lambda, \alpha}$ represents a statistical average for a tagged $\alpha$-isomer, collected along trajectories controlled by the scaled Hamiltonian:

$$
H_{P}^{\mathrm{SC}}(\lambda)=\sum_{i=1}^{N} \sum_{k=1}^{P}\left[\frac{\left(\mathbf{p}_{i}^{(k)}\right)^{2}}{2 M_{i}}+\frac{M_{i} \omega_{p}^{2}}{2}\left(\mathbf{r}_{i}^{(k)}-\mathbf{r}_{i}^{(k+1)}\right)^{2}\right]+V\left(\left\{\tilde{\mathbf{r}}_{i}^{(k)}\right\}\right)
$$

where

$$
\tilde{\mathbf{r}}_{i}^{(k)}=\lambda \mathbf{r}_{i}^{(k)}+(1-\lambda) \mathbf{r}_{i}^{\mathrm{cnt}}
$$

For the pentamers, the latter computational scheme yields

$$
\Delta A_{5 \mathrm{ip}}^{\mathrm{cl} \rightarrow \mathrm{qn}}-\Delta A_{5 \mathrm{p}}^{\mathrm{cl} \rightarrow \mathrm{qn}}=1.0 \pm 0.2 \mathrm{kcal} \mathrm{mol}^{-1}
$$

whereas, for the octamers,

$$
\Delta A_{8 \mathrm{p}}^{\mathrm{cl} \rightarrow \mathrm{qn}}-\Delta A_{8 \mathrm{p}}^{\mathrm{cl} \rightarrow \mathrm{qn}}=1.3 \pm 0.2 \mathrm{kcal} \mathrm{mol}^{-1}
$$

Note that the latter two values compare reasonably well with the free energy differences between the classical and quantum curves at the product states that appear in Fig. 9.

On the other hand, the values of $A_{\alpha}^{\mathrm{SC}}(\lambda=1)$ represent twice the virial estimates of the quantum kinetic energy ${ }^{36}$ of the clusters $\left(\mathrm{KE}_{\alpha}^{\mathrm{qn}}\right)$, in excess of the classical $1.5 N k_{B} T$ expression. Simulation results for $\mathrm{KE}_{\alpha}^{\mathrm{qn}}$ do not differ substantially from the previous free energy differences, namely:

$$
\mathrm{KE}_{5 \mathrm{ip}}^{\mathrm{qn}}-\mathrm{KE}_{5 \mathrm{p}}^{\mathrm{qn}}=1.3 \pm 0.2 \mathrm{kcal} \mathrm{mol}^{-1}
$$

and

$$
\mathrm{KE}_{8 \mathrm{p}}^{\mathrm{qn}}-\mathrm{KE}_{8 \mathrm{p}}^{\mathrm{qn}}=1.4 \pm 0.2 \mathrm{kcal} \mathrm{mol}^{-1}
$$


The latter results would indicate the following: (i) first, a large cancellations between the rest of the contributions, i.e. potential energy and the entropy and (ii) In passing, we remark that, as expected, the increments in the quantum kinetic energies in the product states were also manifest in the $\Delta \mathcal{R}_{\mathrm{H}} \sim 0.05-0.1 \AA$ shrinkages of the correlation lengths of all isomorphic H-polymers (see Eq.(13)) revealing more marked spatial localizations of the light nuclei in clusters exhibiting charge separation.

We finally would like to comment on results from a different kind of simulation runs that bring support to our previous descriptions from a complementary dynamical perspective. Dynamical schemes based on path-integral implementation normally require some degree of approximation. As such, trajectories generated by the Hamiltonian of Eq. (2) correspond to the Ring-PolymerMolecular-Dynamics (RPMD) approach developed by Manolopoulos and collaborators. ${ }^{37}$

We started by considering a set of statistically independent configurations of the $\mathrm{W}_{8 \mathrm{ip}}$ cluster collected along a PIMD canonical trajectory, in which the $\mathrm{O}-\mathrm{H}$ distances in the $\left[\mathrm{H}_{3} \mathrm{O}\right]^{+}$group were maintained close to $1 \AA$ by additional harmonic forces. These configurations were used as initial conditions of a second set of fully unconstrained, microcanonical trajectories, with initial atomic velocities distributed according to Boltzmann statistics. In all cases, charge recombination episodes took place, typically, within the time spans of $\sim 10$ ps. In the two panels of Fig. 10 we present results for the time evolutions of the individual $\xi_{i} \mathrm{~s}$ and $d_{\mathrm{O}_{\mathrm{i}} \mathrm{O}_{\mathrm{i}+1}}$ distances along these runs. To facilitate the comparison, the trajectories appear time ordered, so that all $\xi_{i}$ s for the reactive branches vanish at $t=0$. At a first glance one can observe practically perfect correlation not only between the three different $\xi_{i}$ s along the recombination branches (represented by red lines in the top panel) but also in the other two non-reactive branches, where the individual values of $\xi_{i}$ suffer contractions from $\sim-0.75 \AA$ down to $\sim-1 \AA$ as the recombinations evolve. Similar level of correlations were detected in the changes of the $\mathrm{O}_{\mathrm{i}}-\mathrm{O}_{\mathrm{i}+1}$ distances shown in the lower panel. A more interesting behavior, though, can be seen from the comparison of the sets of red lines in the two panels, where the evolutions of the collective proton transfers go hand in hand with the contractions in the corresponding $\mathrm{O}_{\mathrm{i}}-\mathrm{O}_{\mathrm{i}+1}$ distances. This feature, in turn, would identify collec- 
tive $\mathrm{O}-\mathrm{O}$ compressions as a common correlated mechanism for direct (dissociation) and reverse (recombination) episodes, although the magnitude of the free energy costs of such fluctuations in the former processes are considerably larger than those observed along the latter ones. We remark that similar conclusions were reported in previous ab initio molecular dynamics studies analyzing recombination mechanisms in bulk water ${ }^{4,5}$ and are also consistent with well documented modulation of proton tunneling in ice by the distance between donor/acceptor pairs. ${ }^{38,39}$ Finally, we would like to stress that, although it is well known that the dynamical predictions from RPMD experiments might be subjected to some limitations, ${ }^{37,40}$ we still believe that these observations support the importance of the role of collective proton transfer paths as relevant reaction coordinates in aqueous clusters.

\section{Conclusions}

The computer simulation results presented in this paper provide new insights about combined effects from solvation and nuclear quantum fluctuations on the mechanisms that drive collective proton transfers in small water clusters.

In particular, we focused attention on two, in principle, independent reactive paths described by collective variables involving equally weighted sums of a set of tagged asymmetric stretching coordinates. First, we examined collective proton transfers along cyclic structures in the $\mathrm{W}_{5 \mathrm{p}}$ isomer of the water pentamer and in the $\mathrm{W}_{8 \mathrm{p}}$ isomer of the water octamer, that lead to exchanges of donor/acceptor roles in $\mathrm{HBs}$ between neighboring water molecules. Second, we analyzed collective transfers along chain-like arrangements of water molecules that, starting from the neutral $\mathrm{W}_{5 \mathrm{p}}$ and $\mathrm{W}_{8 \mathrm{p}}$ structures give rise to the $\mathrm{W}_{5 \mathrm{ip}}$ and to the $\mathrm{W}_{8 \mathrm{p}}$ conformers, both exhibiting stable $\left[\mathrm{H}_{3} \mathrm{O}\right]^{+}-$ $[\mathrm{OH}]^{-}$charge separation.

Concerning the first collective mode, the explicit incorporation of nuclear quantum fluctuations transforms the symmetric, bistable profile of the classical free energy into a new one with two distinctive regimes. The first domain, at the inception of the collective transfer, exhibits quadratic 
characteristics. This feature does not differ substantially from the classical result, as it is mostly associated with gradual compressions of distances between much heavier oxygen atoms. As $\xi$ surpasses a threshold value close to $\sim-0.6 \AA$, the plot presents a sharp transition into a second, plateau-like regime where the collective proton transfer is controlled by concerted nuclear tunneling of the light protons. Within the path-integral description, the absence of significant changes observed along the latter regime can be interpreted in terms of the gradual migration of a larger fraction of isomorphic beads between two symmetric basins of attraction at practically no energy costs. As a net result, the magnitude of the resulting quantum free energy barrier is reduced by a factor of $\sim 4$, compared to the one corresponding to the classical description.

The extent of concertedness is altered by the particular features of the coupling between the active ring structures and the rest of the cluster constituents, which act as "solvation baths", with anisotropic spatial characteristics. For the particular case of the $\mathrm{W}_{5 \mathrm{p}}$ pentamer, this coupling involves a single $\mathrm{W}_{\mathrm{B}}$ molecule and promotes a mild global polarization of the 4-ring structure along the direction joining the DDSA and the SDDA water molecules. As such, the different scenarios throughout the reactive path present only minor modifications compared to the ones observed in collective transfers in the absence of coupling such as, for example, in the planar tetramer case, where full correlation prevails. ${ }^{41}$ Contrasting, in the octamer case, the degree of concertedness is somewhat more disparate. In particular, the coupling with two $\mathrm{W}_{\mathrm{B}}$ molecules leads to more marked contractions of the $\mathrm{O}_{\mathrm{i}}-\mathrm{O}_{\mathrm{i}+1}$ distances along the $\mathrm{B}_{1}$ branch compared to those observed along the $\mathrm{B}_{2}$ branch. As a result, configurations corresponding to samplings restricted to the vicinity of $\bar{\xi} \sim-0.6 \AA$, may exhibit mixed characteristics, combining localized (non-tunneling) and delocalized (tunneling) protons along different branches. On the other hand, the more uniform distribution of O-O distances at the transition states reestablishes a higher degree of concertedness between the individual $\left\langle\xi_{i}\right\rangle \mathrm{s}$.

Concerning the second reaction path, the classical analysis of collective transfers along a single branch reveals the presence of solvation induced, $\sim 2-3 \mathrm{kcal} \mathrm{mol}^{-1}$ free energy barriers, stabilizing zwitterionic product states. However, such stabilization is severely deterred as nuclear 
quantum fluctuations are introduced; under these conditions, the free energy barriers are replaced by linear-like, intermediate profiles joining reactant and product states. In a similar way, within a path-integral perspective, these tunneling effects can be easily identified with inter-basin bead migrations, which are manifested in the free energy profile as a population-weighted average of two energy minima of an effective asymmetric double well potential.

Interestingly, the absence of stabilizing barriers in the $\mathrm{W}_{5 i p}$ and $\mathrm{W}_{8 \mathrm{p}}$ clusters opens possibilities to fast recombinations which, given the symmetry characteristics of the HB bonding connectivities, can be driven either along the original reactive branch, or along the other equivalent branches. This establishes a connection between the two reactive channels examined here, as the charge separated product of the second reactive path could be, in principle, also considered as a shortlived intermediate state along the first one.

Before closing this article, we feel important to briefly comment on the possibility of achieving stable charge separation in a water octamer conformer with a different HB connectivity pattern. We are referring to the $\mathrm{W}_{8 \text { cip }}$ cubic arrangement ${ }^{29}$ in which the $\left[\mathrm{H}_{3} \mathrm{O}\right]^{+}$and the $[\mathrm{OH}]^{-}$ionic species lie at the ends of one of the cube diagonals (see Fig. S3 in the Supplemental Information). In fact, we verified that, at $T \sim 50 \mathrm{~K}$, this cluster presents solid-like characteristics, with minimal distortions of its original HB connectivity along all the classical and PIMD trajectories that we investigated. As such, one could speculate on dissociative pathways that, starting with the also stable $\mathrm{C}_{1(g)}$ isomer reported in Ref. [42], and via a collective transfer along three adjacent sides of the cube, would lead to the $\mathrm{W}_{8 \text { cip }}$ conformer. Unfortunately, all our efforts to envisage possible reactive paths involving not only collective, but also sequences of individual H-transfers proved to be unsuccessful, since charge displacements along the perpendicular sides of the clusters ended up promoting a complete loss of the original cubic HB-connectivity. Whether or not this represents a flaw of the performance of the OSS2 Hamiltonian is surely an issue that deserves further investigations which, however, are well beyond the scope of the present paper. 


\section{Supporting Information}

Additional technical details of the simulation procedure are provided in the Supporting Information section.

\section{Acknowledgements}

JR and DL is a staff member of CONICET-Argentina. This work was supported by a research grant to L.T. from the National Research, Development and Innovation Office, Hungary (NKFIH, grant number K128136). The computations were partly performed on the supercomputers of the National Information Infrastructure Development (NIIF) Institute of Hungary. 


\section{References}

(1) Geissler, P. L.; Dellago, C.; Chandler, D.; Hutter, J.; Parrinello, M. Autoionization in Liquid Water. Science 2001, 291, 2121-2124.

(2) Moqadam, M.; Lervik, A.; Riccardi, E.; Venkatraman, W.; Alsberg, B. K.; van Erp, T. S. Local Initiation Conditions for Water Autoionization. Proc. Natl. Acad. Sci. USA 2017, 115, E4569-E4576.

(3) Lockwood, G. K.; Garofalini, S. H. Lifetimes of Excess Protons in Water Using a Dissociative Water Potential. J. Phys. Chem. B 2013, 117, 4089-4097.

(4) Hassanali, A.; Prakas, M. K.; Eshet, H.; Parrinello, M. On the Recombination of Hydronium and Hydroxyde Ions in Water. Proc. Natl. Acad. Sci. USA 2011, 108, 20410-20415.

(5) Lee, S. H.; Rasaiah, J. C. Note: Recombination of $\mathrm{H}^{+}$and $\mathrm{OH}^{-}$ions along water wires. J. Phys. Chem. 2013, 139, 036102.

(6) Trout, B. L.; Parrinello, M. Analysis of the Dissociation of $\mathrm{H}_{2} \mathrm{O}$ in Water Using FirstPrinciples Molecular Dynamics. J. Phys. Chem. B 1999, 103, 7340-7345.

(7) Kattirtzi, J. A.; Limmer, D. T.; Willard, A. P. Microscopic Dynamics of Charge Separation at the Aqueous Electrochemical Interface. Proc. Natl. Acad. Sci. USA 2017, 114, 13374-13379.

(8) Muñoz Santiburcio, D.; Marx, D. Nanoconfinement in Slit Pores Enhances Water SelfDissociation. Phys. Rev. Lett. 2017, 119, 056002.

(9) Perez Serkin, Y. A.; Hassanali, A.; Scherlis, D. A. One-Dimensional Confinements Inhibits Water Dissociation in Carbon Nanotubes. J. Phys. Chem. Lett. 2018, 9, 5029-5033.

(10) Litman, Y.; Donadio, D.; Ceriotti, M.; Rossi, M. Decisive Role of Nuclear Quantum Effects on Surface Mediated Water Dissociation at Finite Temperature. J. Chem. Phys. 2018, 138, 102320. 
(11) Mashaghi, A.; Partovi-Azar, P.; Jadidi, T.; Anvari, M.; Jand, S. P.; Nafari, N.; Tabar, M. R. R.; Maass, P.; Bakker, H. J.; Bonn, M. Enhanced Autoionization of Water at Phospholipic Interfaces. J. Phys. Chem. C 2012, 117, 510-514.

(12) Pinheiro Moreira, P. A. F; de Koning, M. Nuclear Quantum Fluctuations in Ice Ih. Phys. Chem. Chem. Phys. 2015, 17, 24716-24721.

(13) Lee, C.; Sosa, C.; Novoa, J. J. Evidence of the Existence of Dissociated Water Molecules in Water Clusters. J. Chem. Phys. 1995, 103, 4360-4362.

(14) Jensen, J. O.; Samuels, A. C.; Krishnam, P. N.; Burke, L. A. Ion Pair Formation in Water Clusters: a Theoretical Study. Chem. Phys. Lett. 1997, 276, 145-151.

(15) Cárdenas, R.; Lagúnez-Otero, H.; Flores-Rivero, A. Ab Initio Study of the Reaction Mechanism of Water Dissociation into the Ionic Species $\mathrm{OH}^{-}$and $\mathrm{H}_{3} \mathrm{O}^{+}$. Int. J. Quant. Chem. 1998, $68,253-259$.

(16) Tozer, D. J.; Lee, C.; Fitzgerald, An Investigation of Hydrogen Transfer in Water Clusters. J. Chem. Phys. 1996, 104, 5555-5557.

(17) Drechsel-Grau, C.; Marx, D. Exceptional Isotopic-Substitution Effect: Breakdown of Collective Proton Tunneling in Hexagonal Ice due to Partial Deuteration. Angew. Chem. Int. Ed. 2014, 53, 10937-10940.

(18) Drechsel-Grau, C.; Marx, D. Collective Proton Transfer in Ordinary Ice: Local Environments, Temperature Dependence and Deuteration Effects. Phys. Chem. Chem. Phys. 2017, $19,2623-2635$.

(19) Feng, X.; Wang, Z.; Guo, J.; Chen, J.; Wang, W.-G.; Jiang, Y.; Li, X.-Z. The Collective and Quantum Nature of Proton Transfer in the Cyclic Water Tetramer on $\mathrm{NaCl}(001)$. J. Chem. Phys. 2018, 148, 102329.

(20) Throughout this paper, we have used cluster notations similar to those appearing in Ref. [29]. 
(21) The average dihedral angles obtained at the intersections of planes containing different branches were, in all cases, $120^{\circ} \pm 2^{\circ}$.

(22) Tuckerman, M. E. and Hughes, A., in Classical and Quantum Dynamics in Condesed Phase Simulations, edited by B. J. Berne, G. Ciccotti and D. F. Coker (World Scientific, Singapore, 1998) Chap. 14.

(23) Chandler, D.; Wolynes, P. G. Exploiting the Isomorphism between Quantum Theory and Classical Statistical Mechanics of Polyatomic Fluids. J. Chem. Phys. 1081, 74, 4078-4095.

(24) Habershon, S.; Fanourgakis, G. S.; Manolopoulos, D. E. Comparison of Path Integral Molecular Dynamics Methods for the Infrared Absortion Spectrum of Liquid Water. J. Chem. Phys. 2008, 129, 74501.

(25) Tuckerman, M.; Berne, B. J.; Martyna, G. J. Reversible Multiple Time Scale Molecular Dynamics. J. Chem. Phys. 1992, 97, 1990-2001.

(26) Drechsel-Grau, C.; Marx, D. Qantum Simulation of Collective Proton Tunneling in Hexagonal Ice Crystals. Phys. Rev. Lett. 2014, 112, 148302.

(27) Martyna, G. J.; Tuckerman, M. E.; Tobias, D. J.; Klein, M. L. Explicit Reversible Integrators for Extended Systems Dynamics. Mol. Phys. 1996, 87, 1117-1157.

(28) Ceriotti, M.; Parrinello, M.; Markland, T. E.; Manolopoulos, D. E. Efficient Stochastic Thermostatting of Path Integral Molecular Dynamics. J. Chem. Phys. 2010, 133, 124104.

(29) Perlt, E.; von Domaros, M.; Kirchner, B.; Ludwig, R.; Weinhold, F. Predicting the Ionic Product of Water. Sci. Rep. 2017, 7, 20144.

(30) Ojamäe, L.; Shavitt, I.; Singer, S. J. Potential Models for Simulations of the Solvated Proton in Water. J. Chem. Phys. 1998, 109, 5547-5564.

(31) Cendagorta, J. R.; Powers, A.; Hele, T. J. H.; Marsalek, Z., O. Bačić; Tuckerman, M. E. Competing Quantum Effects in the Free Energy Profiles and Diffusion Rates of Hydrogen 
and Deuterium Molecules through Clathrate Hydrates. Phys. Chem. Chem. Phys. 2016, 28, 32169-32177.

(32) Frenkel, D.; Smit, B. Understanding Molecular Simulation. From Algorithms to Applications; Academic Press, 2001; Chapter 7.

(33) Nichols, A. L.; Chandler, D.; Singh, Y.; Richardson, D. M. Excess Electrons in Simple Fluids. II. Numerical Results for the Hard Sphere Solvent. J. Chem. Phys. 1984, 81, 5109.

(34) Videla, P. E.; Rossky, P. J.; Laria, D. Nuclear Quantum Effects on the Structure and the Dynamics of $\left[\mathrm{H}_{2} \mathrm{O}\right]_{8}$ at Low Temperatures. J. Chem. Phys. 2013, 139, 174315.

(35) Habershon, S.; Manolopoulos, D. E. Thermodynamic Integration from Classical to Quantum mechanics. J. Chem. Phys. 2011, 135, 224111.

(36) Herman, M. F.; Bruskin, J.; Berne, B. J. On Path Integral Monte Carlo Simulations. J. Chem. Phys. 1982, 76, 5150-5155.

(37) Habershon, S.; Manolopoulos, D. E.; Markland, T. E.; Miller III, T. F. Ring-Polymer Molecular Dynamics: Quantum Effects in Chemical Dynamics from Classical Trajectories in an Extended Phase Space. Annu. Rev. Phys. Chem. 2013, 64, 387.

(38) Benoit, M.; Marx, D.; Parrinello, M. The Role of Quantum Effects and Ionic Defects in High-Density Ice. Solid State Ionics 1999, 1259, $23-29$.

(39) Benoit, M.; Marx, D.; Parrinello, M. The Role of Quantum Effects and Ionic Defects in High-Density Ice. Nature 1998, 392, 258-261.

(40) Ivanov, S. D.; Witt, A.; Shiga, M.; Marx, D. Communications: On Artificial Frequency Shifts in Infrared Spectra Obtained from Centroid Molecular Dynamics: Infrared Spectrum of Liquid Water. J. Chem. Phys. 2010, 132, 031101. 
(41) Meng, X.; Guo, J.; Peng, J.; Chen, J.; Wang, Z.; Shi, J.-R.; Li, X.-Z.; Wang, W.-G.; Jiang, Y. Direct Visualization of Concerted Proton Tunnelling in a Water Nanocluster. Nature Phys. 2015, 11, 235-239.

(42) Belair, S. D.; Francisco, J. S. Stability of the Cubic Water Octamer. Phys. Rev. A 2003, 67, 63206. 


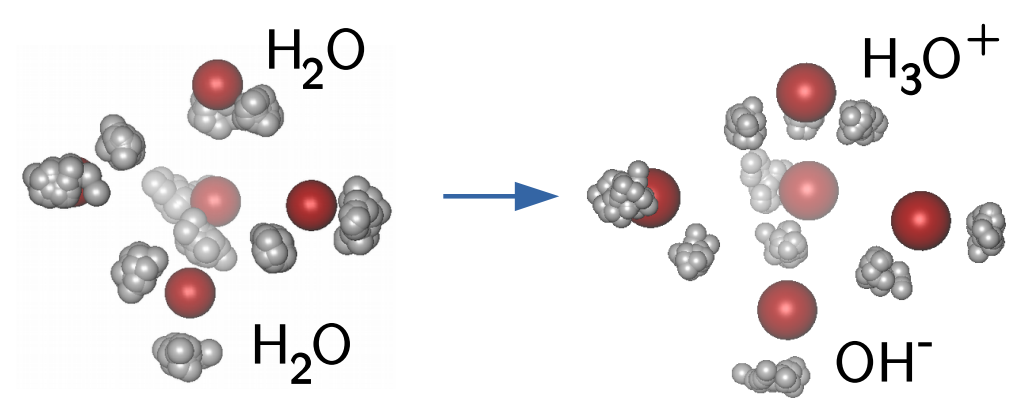

TOC figure

33

ACS Paragon Plus Environment 